\title{
Pratiques
}

Linguistique, littérature, didactique

161-162 | 2014

Écrire/faire écrire

\section{Apprendre à écrire et construire des savoirs explicites sur la langue et la communication par l'étude d'un genre textuel : description de pratiques d'enseignants au niveau primaire}

Teaching of Writing and Knowledge Construction Centred on a Specific Text Genre: Description of Teachers' Practices at Primary Level

\section{Véronique Marmy Cusin}

\section{OpenEdition Journals}

Édition électronique

URL : http://journals.openedition.org/pratiques/2099

DOI : 10.4000/pratiques.2099

ISSN : 2425-2042

Éditeur

Centre de recherche sur les médiations (CREM)

Édition imprimée

Date de publication : 5 juin 2014

Référence électronique

Véronique Marmy Cusin, « Apprendre à écrire et construire des savoirs explicites sur la langue et la communication par l'étude d'un genre textuel : description de pratiques d'enseignants au niveau primaire », Pratiques [En ligne], 161-162 | 2014, mis en ligne le 05 juin 2014, consulté le 19 avril 2019. URL : http://journals.openedition.org/pratiques/2099; DOI : 10.4000/pratiques.2099

Ce document a été généré automatiquement le 19 avril 2019

(c) Tous droits réservés 
Apprendre à écrire et construire des savoirs explicites sur la langue et la communication par l'étude d'un genre textuel : description de pratiques d'enseignants au niveau primaire

Teaching of Writing and Knowledge Construction Centred on a Specific Text Genre: Description of Teachers' Practices at Primary Level

Véronique Marmy Cusin

\section{Introduction}

1 Développer la capacité des élèves à utiliser adéquatement leur langue dans les situations multiples de la vie quotidienne, scolaire ou professionnelle, tout en respectant les normes langagières, est la visée principale de tout enseignement de français. Mais cette double intention (pratique de la langue et respect des normes) pose une question importante pour la recherche en didactique : Que font les enseignants lorsqu'ils ont pour intention de développer la capacité des élèves à écrire et y articulent la construction de connaissances explicites sur la langue et la communication? L'objectif de cet article est de présenter des pratiques d'enseignants au niveau primaire qui tendent vers une telle articulation.

2 Le développement des capacités langagières des élèves et le travail de conceptualisation sur la langue et la communication n'ont pas toujours été liés ; plusieurs courants du $\mathrm{XX}^{\mathrm{e}}$ siècle ont tenté des rapprochements entre ces deux axes de l'enseignement de la langue et cette volonté d'articulation a fait depuis l'objet de nombreuses réflexions et propositions didactiques. En Suisse romande, les orientations actuelles de l'enseignement 
$\mathrm{du}$ français insistent sur ce rapprochement en mettant les textes, et notamment les genres textuels, au centre de l'enseignement du français (CIIP, 2006, 2010). Dans la première partie de cet article, nous définissons plusieurs notions propres à l'articulation entre enseignement de l'écriture et apprentissage grammatical : «Qu'est-ce qu'un genre textuel et comment peut-il être un point d'appui à l'enseignement de l'écriture ? Qu'entendons-nous par articulation entre étude d'un genre textuel et apprentissage grammatical?»

Dans une deuxième partie, nous définissons ce que nous entendons par pratique enseignante puis présentons les caractéristiques méthodologiques d'une recherche doctorale (Marmy Cusin, 2012) centrée sur la pratique d'enseignants mettant en œuvre une séquence didactique créée par la chercheuse et proposant un enseignement articulé de l'écriture et de la grammaire.

4 La troisième partie de cet article présente quelques résultats extraits de cette recherche et analyse deux aspects de la mise en œuvre de la séquence didactique proposée : «Quels sont les gestes fondamentaux des enseignants observés et comment ces enseignants parlent-ils de l'objet grammatical enseigné dans leurs consignes et institutionnalisations?»

\section{Apprendre à écrire et construire des savoirs explicites sur la langue et la communication par l'étude d'un genre textuel}

\section{Comment un genre textuel peut-il être un appui à l'apprentissage de l'écriture?}

5 Si l'on s'intéresse à l'enseignement de la production textuelle, il semble d'abord important de rappeler ce qu'est un texte. J.-P. Bronckart (2004:1) définit le texte comme « la manifestation concrète d'une activité langagière, produite en modalité orale ou écrite, en exploitant les ressources d'une langue donnée (pour ce qui nous concerne, le français) dans le cadre global d'une activité générale déterminée ». Tout texte singulier peut être décrit comme une action langagière singulière au service des activités générales de l'être humain et se déroulant dans un contexte qui lui est propre (Bronckart, 1996b). Il contient un message organisé linguistiquement et vise à produire un but en fonction d'un destinataire (Bronckart, 1996a). Si un texte peut être la manifestation orale ou écrite d'une activité langagière, nous centrons ici notre regard sur l'écriture.

6 En Suisse romande s'est développée l'idée que l'unité « genre textuel » pourrait être un outil didactique pertinent pour apprendre aux élèves à mieux développer la production textuelle et notamment l'écriture. Mais qu'est-ce qu'un genre textuel et comment peut-il devenir un outil au service de l'enseignement de l'écriture? De nombreux auteurs (notamment Combettes, 1988 et Bronckart, 1996) ont mis en évidence qu'un texte n'est pas qu'un assemblage de phrases, mais un tout linguistiquement organisé avec des règles mettant en évidence sa textualité et non sa grammaticalité. Une analyse linguistique des textes définit différents types de productions verbales identifiables dans les pratiques langagières. Ces types de productions verbales sont des sortes de modèles sur lesquels tout locuteur se base pour construire sa propre activité langagière. Pour Bronckart (1996, 2004), ces différentes sortes de textes présentent des caractéristiques relativement 
stables, justifiant qu'ils soient qualifiés de genres de textes en référence à Bakhtine. Lorsqu'un locuteur écrit ou parle, il ne crée jamais quelque chose de totalement nouveau, du moins du point de vue de la structure du texte. Il s'appuie sur les textes déjà existants socialement et historiquement dans l'intertexte pour construire son propre texte. Il choisit le genre textuel le plus adapté à la situation de production telle qu'il se la représente et adapte le modèle choisi aux particularités de cette situation. Et ce processus d'adaptation conduit à terme à une modification des genres textuels disponibles dans l'intertexte (Bronckart, 2004). Par genre textuel, nous entendons donc toute forme textuelle suffisamment stable et partagée pour que les locuteurs la reconnaissent comme un support stable de l'activité langagière. Le conte, la lettre au courrier des lecteurs, la recette de cuisine ou le fait divers sont des genres textuels.

7 Si tout texte est par définition différent, un genre textuel suit certaines règles d'organisation communes (Bronckart, 1996, 2004): son infrastructure générale (notamment l'utilisation et l'emboitement de différentes séquences textuelles), ses règles de textualisation qui visent l'unité et la cohérence du texte et ses mécanismes de prise en charge énonciative. Partant de ce constat, B. Schneuwly et J. Dolz (1997 : 39) développent l'hypothèse selon laquelle "la définition aussi précise que possible des dimensions enseignables d'un genre facilite l'appropriation de celui-ci et rend possible le développement de capacités langagières diverses qui y sont associées ». Le genre peut donc devenir un outil au service des enseignants et de leurs élèves. L'élaboration de moyens romands pour développer l'expression orale et écrite (Dolz et al., 2001) s'est faite à partir de cette unité significative du genre ; ces moyens offrent des outils concrets pour développer certaines opérations propres à l'écriture d'un texte et pour travailler certains phénomènes de textualisation en lien étroit avec des situations de communication réelles. Travailler à partir d'un genre, c'est donc l'occasion de découvrir et de s'approprier les caractéristiques d'un genre textuel particulier tout en donnant du sens à des apprentissages langagiers spécifiques que nous appelons apprentissages grammaticaux. En effet, si l'enseignement grammatical concerne traditionnellement l'étude d'une partie des systèmes et sous-systèmes de la langue, il s'est élargi, sous l'influence de certaines théories liées à la grammaire textuelle et à l'énonciation, à certains phénomènes de textualisation (segmentation textuelle et connexion, cohérence nominale et verbale) et d'énonciation (emploi de déictiques, modalisation, discours rapportés) (Chartrand \& Boivin, 2004). Nous optons donc pour le terme d'enseignement grammatical dans la suite de ce texte pour parler de tout enseignement explicite sur la langue et la communication, même si nous sommes bien consciente que les phénomènes de textualisation et d'énonciation sont des notions métalinguistiques fort différentes de celles généralement étudiées en morphosyntaxe (Maingueneau, 2005); elles sont notamment plus difficiles à formaliser de manière claire et définitive. Nous pensons cependant qu'il est essentiel de construire des connaissances explicites sur ces phénomènes afin d'éviter un apprentissage uniquement intuitif de la langue par une utilisation de celle-ci en contexte, qui « doit peu à l'enseignement de l'école, et beaucoup au milieu familial d'origine » (Maingueneau, 2005 : 54). 


\section{Qu'entendons-nous par articulation entre étude d'un genre textuel et apprentissage grammatical ?}

De nombreux didacticiens (notamment Chartrand, 1996 ; Tisset \& Léon, 1992) proposent de mettre en lien apprentissage grammatical et pratiques langagières; ces auteurs soulignent que l'objectif principal de l'enseignement de la grammaire à l'école est la maitrise de normes, dans la perspective de développer des compétences en compréhension et en production orale et écrite; néanmoins, la plupart des auteurs ne prônent pas un enseignement grammatical dissous dans les activités langagières (Chabanne, 2004).

Dans la perspective de mettre en lien langue-outil de communication et langue-objet grammatical, nous pensons que l'étude de genres textuels particuliers peut servir de cadre à un enseignement structuré de l'écriture en y articulant des savoirs grammaticaux. Par l'étude d'un genre spécifique, il est possible de sensibiliser les élèves aux mécanismes de fonctionnement de ce genre textuel et de conceptualiser certaines notions et règles de planification et de textualisation qui lui sont propres. Dans le cadre de ce travail spécifique, il est aussi possible d'intégrer des apprentissages liés à la syntaxe, au lexique, à l'orthographe grammaticale ou à la conjugaison, en lien avec les objets textuels travaillés.

10 La séquence didactique (voir figure 1) présentée aux enseignants dans le cadre d'une thèse de doctorat (Marmy Cusin, 2012) s'inscrit dans cette perspective ; elle propose un enseignement articulé de la production textuelle et de la grammaire, en faisant des liens explicites entre l'étude d'un genre particulier (le récit d'aventure) et un phénomène de textualisation, les manières de reprendre les personnages au fil du récit (cohésion nominale).

Figure 1. Schéma de la séquence didactique proposée.

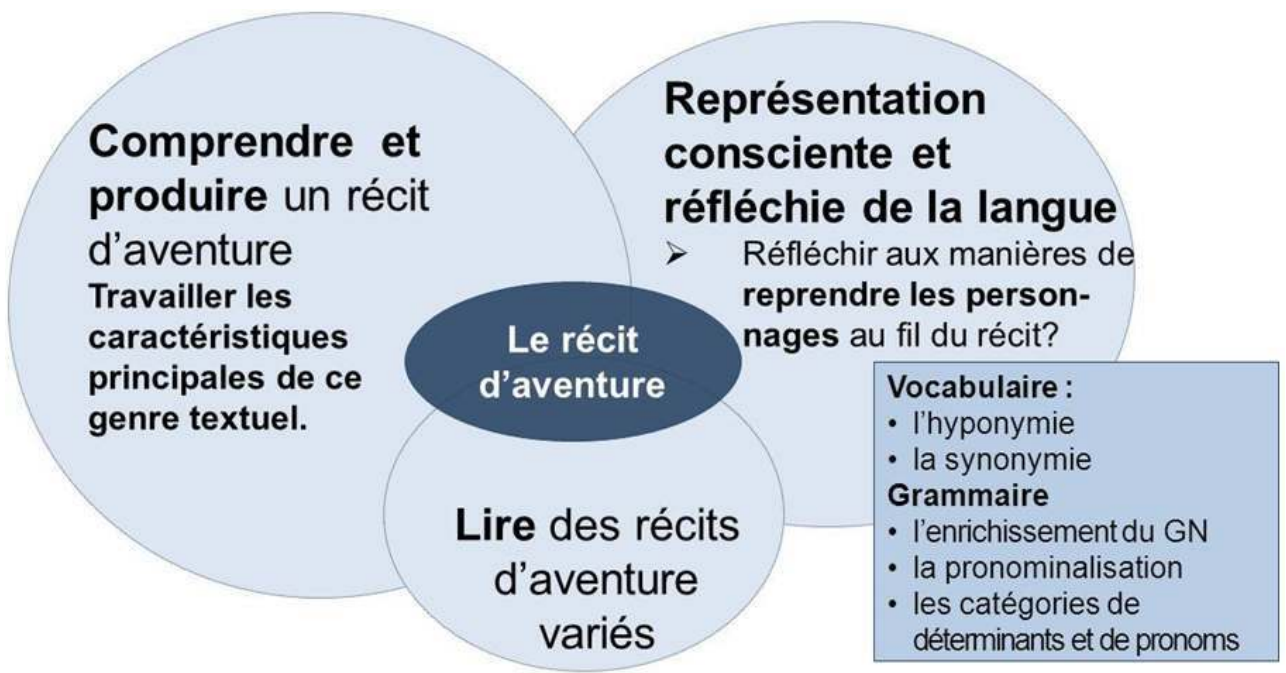

11 La séquence proposée part de l'étude d'un genre particulier, le récit d'aventure, et y articule de multiples activités centrées sur les manières de reprendre les personnages au fil du récit. Précisons que cet objet textuel est relativement nouveau et peu enseigné au niveau primaire au moment de la recherche ${ }^{1}$. De ce fait, la séquence didactique propose davantage une sensibilisation à la manière de reprendre les personnages au fil du récit en 
se centrant sur trois dimensions de l'objet définies par D. Bucheton (1994) et reprises dans notre carte conceptuelle (annexe 3) : dimension textuelle et sémantique, dimension liée au système de la langue et dimension stylistique. Ce travail sur la langue et la communication permet de sensibiliser les élèves à la cohésion nominale et de construire quelques connaissances sur la typologie des reprises utilisées, mais aussi de consolider certaines notions, en faisant des liens étroits avec certaines notions grammaticales (l'enrichissement $\mathrm{du}$ groupe nominal, la pronominalisation, les catégories de déterminants et de pronoms) et lexicales (liens d'hyponymie et de synonymie). Précisons encore que si notre recherche s'est centrée spécifiquement sur les leçons travaillant la cohésion nominale, les enseignants ont aussi approfondi d'autres aspects propres au genre textuel travaillé (séquence narrative, description d'un lieu, discours direct, cohésion verbale).

La partie «Présentation des résultats » décrit certains aspects des pratiques observées dans la mise en œuvre d'une telle séquence didactique. Mais de quelle pratique enseignante parle-t-on et comment la décrire?

\section{Une recherche centrée sur la description de pratiques dans l'enseignement de l'écriture}

\section{Les gestes didactiques au cœur de la pratique enseignante}

13 La pratique enseignante peut se définir comme la façon de faire singulière d'un acteur, l'exécution personnelle de son activité professionnelle. Mais celle-ci ne peut se résumer aux actes observables. Les choix réalisés avant, pendant ou après l'action, les prises de décision sont partie intégrante de cette pratique de l'enseignant (Clot, Faïta \& Scheller, 2000 ; Goigoux, 2007). Si, dans le cadre de notre thèse de doctorat, nous nous sommes intéressée autant aux actes des enseignants qu'à leur discours pour expliquer leur action, dans le cadre de cet article $^{2}$, pour des raisons d'espace, nous circonscrivons notre présentation aux actes de l'enseignant, par l'observation de sa pratique in situ.

Notre regard porte spécifiquement sur l'action didactique au sein de la salle de classe, définie par B. Schneuwly et J. Dolz (2009: 31) comme la transformation « des modes de penser, de parler et d'agir » des apprenants « à l'aide d'outils sémiotiques » et en fonction des finalités définies par le système scolaire. Pour éclairer cette dimension de la pratique enseignante, nous prenons prioritairement appui sur le travail réalisé par le GRAFE (Groupe de recherche pour l'analyse du français enseigné, Schneuwly \& Dolz, 2009). Si plusieurs auteurs ont tenté de définir ce savoir-agir par des termes différents, par exemple «jeu didactique» pour G. Sensevy (2007), ou schèmes professionnels pour R. Goigoux (2007), nous préférons la notion de geste didactique (Schneuwly \& Dolz, 2009), terme qui renvoie aussi bien à la gestuelle et au corps qu'à un savoir-faire professionnel. Le mot geste est bien lié au corps, au mouvement et à l'idée d'atteindre un but. Les gestes s'inscrivent donc dans le corps du sujet et s'ils peuvent être inconscients, ils témoignent souvent d'une intention du sujet, qui, par les gestes utilisés, donne une signification précise à son action. Nous ciblons ici notre analyse sur trois gestes didactiques, conceptualisés par Schneuwly et Dolz (2009) pour observer comment l'enseignant met en scène l'objet d'étude et guide l'attention de l'élève :

- la mise en place de dispositifs didactiques permet à l'enseignant, par des outils autant langagiers (consignes), non langagiers (gestes, déplacements, emplacement dans l'espace) que 
matériels, de présenter l'objet enseigné et de définir ce que l'élève est censé faire (activités scolaires), sa place et son rapport à l'objet enseigné ;

- par le geste de régulation des apprentissages, l'enseignant adapte les dispositifs didactiques mis en œuvre et reconfigure son action en fonction d'observations et de la prise en compte de facteurs multiples. La régulation des apprentissages sert de contrôle et contribue à « confirmer ou réorienter la trajectoire de l'action, et/ou redéfinir le but » (Allal, $2007: 8$ ). Cette régulation interactive s'observe particulièrement à travers l'analyse des interactions entre enseignants et élèves et des outils utilisés (langagiers et non langagiers) pour aider l'élève à construire l'objet d'études. Nous soulignons cependant avec L. Allal que, comme tout geste, cet acte de régulation peut être plus ou moins conscientisé ;

- Le troisième geste retenu est celui de l'institutionnalisation. Pour Schneuwly et Dolz (ibid.), institutionnaliser, c'est fixer de manière explicite et conventionnelle le statut du savoir en construction. De la description de G. Brousseau (1998 : 92), nous pouvons retenir la mise en lien par l'enseignant des actions et productions des élèves et un savoir plus scientifique. Ce même auteur décrit différentes actions observées chez les enseignants durant cette phase d'institutionnalisation :

- $\circ$ s'arrêter pour synthétiser le travail réalisé avant de poursuivre,

- décrire le travail en cours pour le mettre en lien avec «la connaissance visée » et des savoirs plus formalisés,

- donner un «statut aux événements de la classe, comme résultat des élèves et comme résultat de l'enseignant » (ibid. : 311),

- rapprocher ces productions des élèves à des « connaissances des autres (culturelles ou du programme)",

- mettre en évidence à quoi ces connaissances peuvent servir.

Ce temps d'institutionnalisation prend souvent la forme d'un « arrêt, de suspension ou de parenthèse dans l'avancement du temps didactique » (Schneuwly \& Dolz, 2009: 97). Ce geste d'institutionnalisation semble particulièrement important dans la construction de l'objet enseigné : par ce geste, l'enseignant fait comprendre à ses élèves l'objet de l'apprentissage et l'enjeu de celui-ci.

Ces différents gestes, essentiels au travail didactique de l'enseignant, s'inscrivent souvent dans un rapport de temporalité (Schneuwly \& Dolz, 2009). Le travail démarre généralement par une consigne et la mise en place de dispositifs didactiques; il se poursuit par des temps de régulation des apprentissages et se termine par une institutionnalisation du savoir construit; le geste de mémoire didactique, geste non pris en compte dans le cadre de cette recherche, sert de maillage à cette temporalité et apparait tout au long du processus. Néanmoins, «ce rapport temporel n'a rien d'automatique", les consignes pouvant faire l'objet d'une reformulation et l'institutionnalisation pouvant ponctuer l'apprentissage, et non pas intervenir à la toute fin (ibid. : 41).

\section{Une méthodologie de recherche centrée sur la pratique enseignante}

Sous la forme d'une formation continue, il a été proposé à quatre enseignants du deuxième cycle primaire ${ }^{3}$ un dispositif d'ingénierie sous la forme d'une séquence didactique décrite précédemment et de quatre rencontres entre participants. Pour le recueil des données, nous avons analysé les pratiques effectives dans la salle de classe. Pour ce faire, nous avons filmé toutes les leçons spécifiquement centrées sur l'objet 
grammatical imposé, la cohésion des chaines référentielles désignant les personnages du récit (généralement trois leçons entre 50 et 70 minutes par enseignant; environ 700 minutes de pratiques filmées). Ces leçons ont été intégralement transcrites.

Nous centrons notre analyse sur les trois premiers gestes définis par Schneuwly et Dolz (2009) : la mise en place de dispositifs didactiques, la régulation des apprentissages et le geste d'institutionnalisation. Ces analyses ont permis de donner des éléments de réponses aux deux questions de recherche suivantes:

- Que fait l'enseignant lorsqu'il met en œuvre un enseignement articulant écriture et savoirs sur la langue : Quels sont les dispositifs réellement mis en œuvre, les dispositifs régulés et les obstacles identifiés, les institutionnalisations observées?

- Quel est l'objet réellement enseigné (cohésion nominale) visible dans les consignes et institutionnalisations observées au cours de l'action didactique?

\section{Les gestes fondamentaux de l'enseignant quand il articule écriture et savoirs sur la langue}

\section{Les textes au cœur des dispositifs mis en œuvre par les enseignants}

Nous avons analysé les dispositifs ${ }^{4}$ ayant pour objet la cohésion nominale et mis en œuvre par les participants ; ceux-ci ont dû faire des choix parmi les dispositifs proposés dans la séquence didactique.

Tableau 1. Comparaison entre les types de dispositifs proposés dans la séquence didactique et les types de dispositifs visibles dans les quatre pratiques observées.

\begin{tabular}{|l|l|l|l|l|}
\hline Type de dispositifs & $\begin{array}{l}\text { N. de consignes } \\
\text { proposées dans la } \\
\text { séquence }\end{array}$ & $\begin{array}{l}\text { N. total de } \\
\text { dispositifs }\end{array}$ & $\begin{array}{l}\text { N. } \\
\text { mettant ens. } \\
\text { euvre } \\
\text { dispositif }\end{array}$ & $\begin{array}{l}\text { Durée } \\
\text { cetale en } \\
\text { minutes }\end{array}$ \\
\hline $\begin{array}{l}\text { Dispositifs proposant la } \\
\text { lecture et l'analyse de } \\
\text { textes d'auteurs }\end{array}$ & 6 & 27 & $4 / 4$ & 200 \\
\hline $\begin{array}{l}\text { Dispositifs visant l'analyse } \\
\text { de productions textuelles }\end{array}$ & 6 & 21 & $4 / 4$ & 240 \\
\hline $\begin{array}{l}\text { Dispositifs visant la } \\
\text { production d'outils }\end{array}$ & 2 & 11 & $4 / 4$ & 60 \\
\hline $\begin{array}{l}\text { Dispositifs visant une } \\
\text { production textuelle }\end{array}$ & 5 & 6 & $3 / 4$ & - \\
\hline $\begin{array}{l}\text { Dispositifs visant un } \\
\text { apprentissage } \\
\text { grammatical ou lexical } \\
\text { spécifique }\end{array}$ & 5 & - & - & \\
\hline
\end{tabular}




\begin{tabular}{|l|l|l|l|l|}
\hline Total & 24 & 65 & $4 / 4$ & 680 \\
\hline
\end{tabular}
$(21 / 65)$. Cette fréquence élevée est en contradiction avec plusieurs recherches à ce propos. Par exemple, dans une évaluation des moyens officiels romands (De Pietro et al., 2009), les enseignants nomment, comme difficulté principale, l'évaluation des productions initiales des élèves. Pourquoi les enseignants observés ici analysent-ils de manière prépondérante les productions initiales avec leurs élèves alors que cette analyse est habituellement peu mise en œuvre parce que jugée trop difficile? Nous émettons l'hypothèse selon laquelle la séquence didactique, centrée sur un seul phénomène grammatical (la cohésion des chaines référentielles désignant les personnages du récit), a permis de focaliser l'attention des élèves et des enseignants sur cet objet. Cette centration sur un objet textuel spécifique a peut-être aidé les enseignants à accompagner les élèves dans l'observation et l'analyse de leur propre production.

Dernier constat quant aux dispositifs mis en œuvre: aucun participant n'approfondit spécifiquement un objet grammatical ou lexical (par exemple, la synonymie ou les constituants du groupe nominal). Les enseignants observés mettent en œuvre des activités métalinguistiques riches et intéressantes mais toujours liées à la production textuelle, à la production d'outils (par exemple : la recherche de synonymes adaptés au contexte, la construction de groupes nominaux variés pour reprendre le personnage) ou à l'analyse d'un texte d'auteur (par exemple: le répertoire et classement des reprises 
utilisées par un auteur). Ce constat nous invite à penser que lorsque l'analyse grammaticale s'intègre à la production d'un texte, elle se met au service de celui-ci et la construction de savoirs sur la langue n'a pas un statut en soi.

\section{Réguler les apprentissages : une place importante au détriment de l'institutionnalisation des connaissances}

La figure 2 présente les gestes identifiés dans les quatre pratiques observées lors des leçons travaillant la cohésion nominale. Nous observons que le geste de régulation interactive (Allal, 2007) fait partie du fil de l'action enseignante et apparait tout au long de la construction de l'objet grammatical alors que l'institutionnalisation des connaissances en est, au contraire, le " parent pauvre ».

La catégorie « régulation des apprentissages » (figure 2, en vert) répertorie les actions de l'enseignant face à des obstacles nommés par les élèves ou identifiés par l'enseignant luimême.

Figure 2. Gestes observés dans les quatre pratiques singulières ${ }^{5}$.

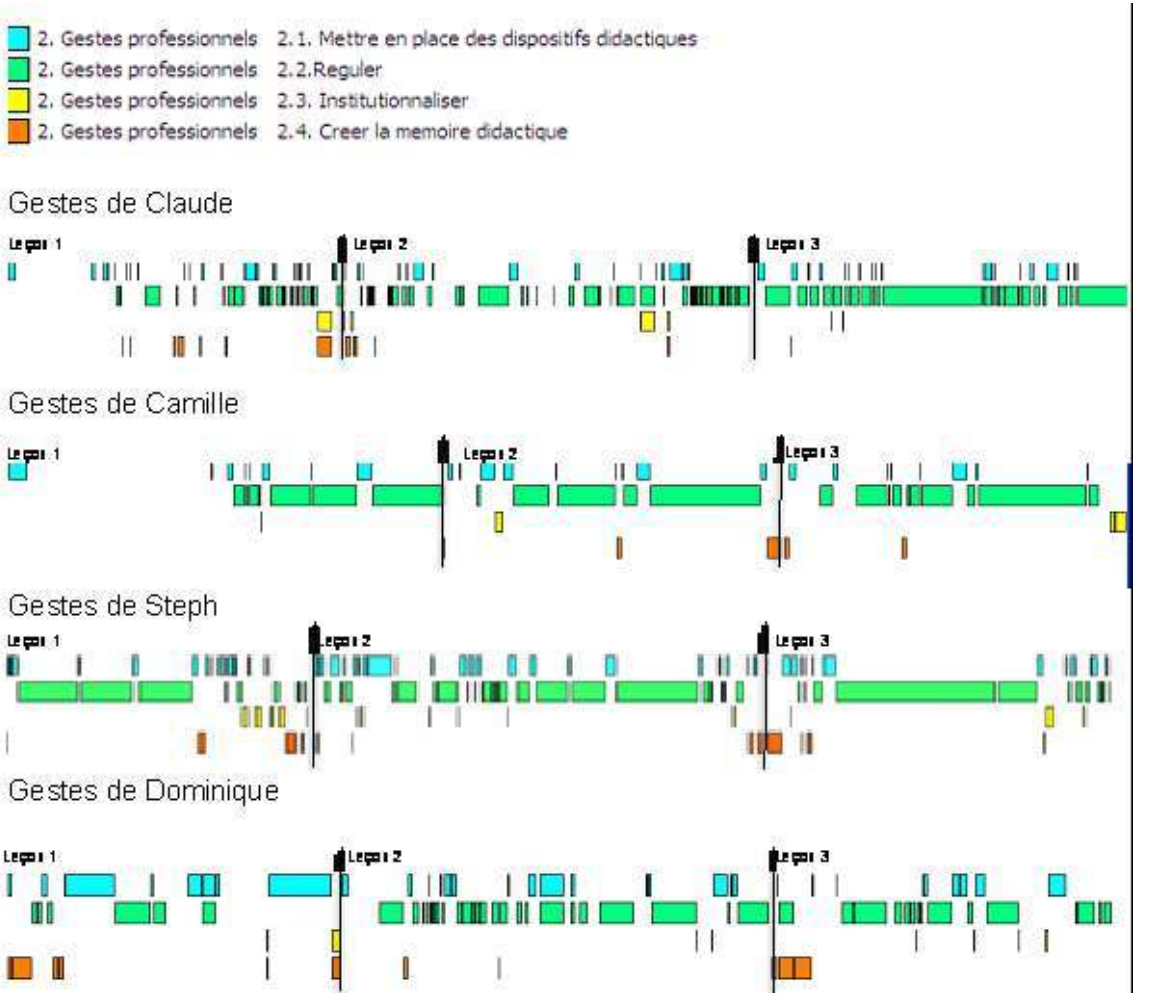

De manière plus spécifique, le tableau 2 répertorie les régulations identifiées en fonction des types de dispositifs.

Tableau 2. Types de dispositifs les plus régulés.

\begin{tabular}{|l|l|l|l|}
\hline Description des dispositifs régulés & $\begin{array}{l}\text { N. de dispositifs } \\
\text { observés }\end{array}$ & $\begin{array}{l}\text { N. d'événements } \\
\text { régulatifs }\end{array}$ & $\begin{array}{l}\text { N. } \\
\text { d'ens. }\end{array}$ \\
\hline
\end{tabular}




\begin{tabular}{|l|l|l|l|}
\hline $\begin{array}{l}\text { Identification et classification des reprises } \\
\text { utilisées dans des textes d'auteurs ou des } \\
\text { productions d'élèves }\end{array}$ & 23 & 21 & $4 / 4$ \\
\hline $\begin{array}{l}\text { Production de reprises variées à l'oral ou à l'écrit } \\
\text { et construction d'outils }\end{array}$ & 13 & 22 & $4 / 4$ \\
\hline $\begin{array}{l}\text { Lecture et compréhension d'un texte aux reprises } \\
\text { particulières } \\
\text { Identification du style de cohérence nominale } \\
\text { utilisé par un auteur }\end{array}$ & 13 & 11 & $4 / 4$ \\
\hline Autres dispositifs régulés (de différentes natures) & 16 & 13 & $4 / 4$ \\
\hline Total & 65 & 67 & $4 / 4$ \\
\hline
\end{tabular}

Ce tableau montre que les dispositifs les plus régulés sont l'identification et la classification des reprises. Ceci peut s'expliquer par le fait que la cohésion nominale est une notion peu connue des élèves et nécessite une médiation; par ailleurs, les textes (d'élèves, d'auteurs) mettent les élèves face à des reprises complexes, parfois difficiles à identifier ou analyser (pronoms personnels compléments de verbe, identification de l'ensemble du groupe nominal utilisé comme reprise).

Afin d'illustrer ce geste, voici un exemple de régulation observée dans une activité d'identification des reprises des personnages du texte « Cœur de lion » (annexe 1).

«Ce que vous faites là/ c'est correct/ mais je pense que ça vaudra la peine/ de relire et puis de vous arrêter sur tous les mots qui désignent un personnage/ là vous allez au repérage/ en cherchant/fourmi/grenouille/ les personnages qu'on a mis au tableau/ mais on a remarqué/ que parfois/ on parle de ces personnages/ sans dire/ couleuvre/ sans dire la grenouille/ certains ont dit d'autres mots qui ont été utilisés avant/ ces mots/ il faut aussi les mettre en évidence/ mais pour ça/ ça vaut la peine de vraiment lire/ pas forcément du repérage de mots/»(Dominique, degré $5^{\mathrm{H}}$, leçon 2).

31 On remarque dans cet extrait que l'obstacle identifié par l'enseignant est lié à la notion de « reprise anaphorique». Plusieurs fois, les enseignants observés ont dû redéfinir avec d'autres mots ce qu'ils entendaient par reprise anaphorique et distinguer ces reprises des autres aspects propres au personnage, telles sa description, ses actions ou ses paroles (dialogues).

32 En ce qui concerne le geste d'institutionnalisation (figure 2, en jaune), il est très peu présent dans les différentes pratiques observées. Afin de répertorier le geste d'institutionnalisation, nous avons pris en compte trois caractéristiques formelles, définies par Schneuwly et Dolz (2009), qui contribuent à mieux comprendre ce geste didactique: idée de généralisation, marques de modalisation/prescriptions, décontextualisation d'un savoir émergent vers un savoir collectif et recontextualisation de ce savoir dans de nouvelles situations. Nous avons décidé de prendre en compte tous les moments au cours desquels l'enseignant mettait en œuvre une de ces trois actions sans nécessairement que chaque institutionnalisation ait les trois caractéristiques simultanément. Nous avons fait ce choix pour deux raisons principales : d'une part, les trois caractéristiques nommées ci-avant restent une définition théorique de 
l'institutionnalisation qui devait être mis à l'épreuve des données elles-mêmes; d'autre part, les institutionnalisations restent peu fréquentes dans notre corpus; de ce fait, nous voulions mettre en évidence chaque moment où l'enseignant va dans la direction d'une institutionnalisation, même si celle-ci peut être considérée comme partielle.

Les institutionnalisations observées sont souvent très courtes et surtout visibles en cours et en fin de leçon pour synthétiser le savoir et lui donner un caractère de généralisation. Les enseignants accompagnent régulièrement cette généralisation d'outils langagiers modalisants pour montrer l'importance du savoir en train d'être construit. Presque la moitié des institutionnalisations observées permettent à l'enseignant de recontextualiser le savoir construit dans une tâche d'écriture future.

Voici deux exemples d'institutionnalisation des connaissances par l'enseignant, suite à la lecture du texte « Le piège » de Yack Rivais (annexe 2) :

« voilà/ le problème c'est que/ lorsqu'on utilise/ seulement des pronoms comme il ou elle/. à nous lecteurs/ ça ne nous dit rien/ ou presque rien/ sur/ les caractéristiques du héros/on sait juste si c'est masculin ou féminin/. mais à part ça/ on sait pas grand chose de plus/ donc on avait découvert que c'était important de/ trouver des moyens/ de donner des informations au lecteur/. et/. d'éviter de répéter/plusieurs fois/ les ils ils/ la maitresse/ la maitresse/ la maitresse/ parce que c'est/ aussi une question de style/ hein/ c'est pas très beau/ d'écrire comme ça/ donc on avait dit que le vocabulaire/ n'est pas très varié/ non plus hein/ donc c'est mieux si on peut enrichir le vocabulaire/ » (Claude ; degré $7-8^{\mathrm{H}}$, leçon 2).

« donc là/ l'auteur/ Yack Rivais/ il a fait exprès/ d'utiliser la recette opposée de ce que/ nous on aimerait/. c'est/ il a utilisé/ que des pronoms/. que des mots/très vagues/ qui désignent très peu les personnes/ pour laisser le suspense/ jusqu'au bout/. mais dans votre récit d'aventure/. l'idée/ c'est d'utiliser la recette opposée/. pour qu'on essaie/ d'avoir des renseignements/. pis de savoir/ où on en est/. ok/ " (Steph ; degré 5-6 ${ }^{\mathrm{H}}$, leçon 2).

Dans les extraits ci-dessus, les enseignants pointent le savoir en construction et tentent une généralisation, caractéristique propre au geste d'institutionnalisation; le premier enseignant utilise aussi des outils modalisants et prescriptifs pour insister sur le style de reprises attendu; le deuxième extrait montre le passage entre des savoirs en construction et leur utilisation future en situation de production textuelle.

\section{L'objet enseigné dans les consignes et institutionnalisations : une visée en premier lieu normative et stylistique}

Notre seconde question de recherche est centrée sur les caractéristiques de l'objet enseigné (cohésion nominale) dans les consignes et institutionnalisations. L'enseignant se réapproprie les savoirs à enseigner et les transpose de manière singulière dans les interactions vécues au cours de l'action; les consignes et institutionnalisations constituent donc un moyen efficace pour pointer cette transposition interne.

Nous avons classé les consignes et institutionnalisations identifiées à l'aide de trois dimensions de l'objet " cohésion nominale », dimensions proposées par Bucheton (1994) et reprises dans notre carte conceptuelle (annexe 3 ) : la dimension ayant trait à des objectifs textuels et sémantiques, la dimension insérant les reprises anaphoriques dans le système de la langue et la dimension abordant les reprises anaphoriques d'un texte d'un point de vue stylistique. 


\section{La prédominance de la dimension textuelle et sémantique de la cohésion nominale dans les consignes et institutionnalisations} variées ou de les utiliser pour améliorer les productions initiales (12/66). Cette idée de varier les reprises choisies pour désigner les personnages, que ce soit dans les productions des élèves ou dans des textes d'auteurs, est fréquemment institutionnalisée $(13 / 31)$ par tous les enseignants.

« donc on voit que/ avec des groupes nominaux/. alors des fois/ j'ai transformé un petit peu ce qui était écrit pour pouvoir faire des groupes nominaux/. mais/. là par exemple/ c'était exactement ce qui était écrit/. on voit qu'avec/. on arrive déjà à se faire une sacrée image/ du personnage/» (Steph ; leçon $3 ; 2: 02: 01$ ).

41 Un autre type de consignes, observées à plusieurs reprises (10/49) dans toutes les pratiques, tente de focaliser l'attention de l'élève sur le texte produit initialement et de lui faire prendre conscience des difficultés et stratégies mises en œuvre pour répondre à la consigne d'écriture. D'autres consignes, liées à la compréhension d'un texte d'auteur, ciblent aussi l'attention des élèves sur la construction d'une image du personnage plus ou moins précise en fonction des substituts choisis par l'auteur. Par ces consignes, les enseignants montrent aux élèves que certaines reprises, notamment pronominales, donnent peu d'indications sur le personnage et que, de ce fait, il est nécessaire de leur préférer des reprises riches et variées si l'on veut décrire le personnage au fil du récit à l'aide de ces reprises.

Tableau 3. Dimensions de l'objet enseigné visible dans les consignes et les institutionnalisations.

\begin{tabular}{|l|l|l|l|l|}
\hline \multirow{2}{*}{ Dimensions de l'objet enseigné } & \multicolumn{2}{|l|}{$\begin{array}{l}\text { visibles dans les } \\
\text { consignes }\end{array}$} & $\begin{array}{l}\text { visibles dans les } \\
\text { institutionnalisations }\end{array}$ \\
\cline { 2 - 7 } & $\begin{array}{l}\text { N. de } \\
\text { ens. }\end{array}$ & $\begin{array}{l}\text { N. } \\
\text { consignes }\end{array}$ & N. ens. & N. d'instit. \\
\hline Sur le plan textuel et sémantique & $4 / 4$ & $\mathbf{4 9 / 6 6}$ & $\mathbf{4 / 4}$ & $\mathbf{2 1 / 3 1}$ \\
\hline $\begin{array}{l}\text { Identifier les reprises utilisées pour reprendre } \\
\text { les personnages. }\end{array}$ & $4 / 4$ & $17 / 66$ & - & - \\
\hline $\begin{array}{l}\text { Produire des reprises variées, améliorer le } \\
\text { choix des reprises dans un texte. }\end{array}$ & $4 / 4$ & $12 / 66$ & $4 / 4$ & $13 / 31$ \\
\hline $\begin{array}{l}\text { Prendre conscience des difficultés liées aux } \\
\text { reprises dans un texte produit ou lu. }\end{array}$ & $4 / 4$ & $10 / 66$ & $3 / 4$ & $7 / 31$ \\
\hline
\end{tabular}




\begin{tabular}{|c|c|c|c|c|}
\hline $\begin{array}{l}\text { Construire une image plus ou moins précise du } \\
\text { personnage à l'aide des reprises utilisées. }\end{array}$ & $4 / 4$ & $6 / 66$ & - & - \\
\hline $\begin{array}{l}\text { Comprendre les liens entre la reprise et le } \\
\text { personnage auquel elle se réfère. }\end{array}$ & $2 / 4$ & $3 / 66$ & - & - \\
\hline Comprendre les liens entre les personnages. & $1 / 4$ & $1 / 66$ & - & - \\
\hline $\begin{array}{l}\text { Favoriser le réinvestissement des } \\
\text { connaissances dans une production future. }\end{array}$ & - & |- & $1 / 4$ & $1 / 31$ \\
\hline Sur le plan du système de la langue & $4 / 4$ & $11 / 66$ & $3 / 4$ & $6 / 31$ \\
\hline $\begin{array}{l}\text { Identifier les types de reprises utilisées pour } \\
\text { reprendre les personnages. }\end{array}$ & $4 / 4$ & $7 / 66$ & $2 / 4$ & $3 / 31$ \\
\hline $\begin{array}{l}\text { Identifier la fonction des reprises nominales et } \\
\text { pronominales. }\end{array}$ & $1 / 4$ & $1 / 66$ & - & - \\
\hline $\begin{array}{l}\text { Réviser ses connaissances de différentes } \\
\text { catégories de pronoms et de déterminants. }\end{array}$ & $2 / 4$ & $2 / 66$ & - & |- \\
\hline $\begin{array}{l}\text { Établir des relations de synonymie pour } \\
\text { reprendre un personnage. }\end{array}$ & $1 / 4$ & $1 / 66$ & $1 / 4$ & $2 / 31$ \\
\hline $\begin{array}{l}\text { Préciser la terminologie textuelle pour parler } \\
\text { des reprises. }\end{array}$ & - & - & $1 / 4$ & $1 / 31$ \\
\hline Sur le plan stylistique & $4 / 4$ & $6 / 66$ & $2 / 4$ & $4 / 31$ \\
\hline $\begin{array}{l}\text { Identifier les caractéristiques d'un texte qui } \\
\text { utilisent une reprise ambigüe du/ des } \\
\text { personnage-s. }\end{array}$ & $3 / 4$ & $5 / 66$ & $2 / 4$ & $2 / 31$ \\
\hline $\begin{array}{l}\text { Comparer deux styles (textes d'auteurs dans } \\
\text { l'utilisation des reprises des personnages } \\
\text { (transparence versus ambigüité). }\end{array}$ & $1 / 4$ & $1 / 66$ & $1 / 4$ & $2 / 31$ \\
\hline TOTAL & $4 / 4$ & 66 & $4 / 4$ & 31 \\
\hline
\end{tabular}

Cette même idée est régulièrement institutionnalisée (7/31). Il semble donc important pour ces enseignants de rendre attentifs leurs élèves à l'intérêt de maintenir une certaine clarté dans leurs textes pour favoriser la compréhension du récit par un lecteur externe. Ils centrent aussi l'attention des élèves sur la nécessité d'éviter les ambigüités en préférant des reprises précises et claires quant au personnage " référent ».

Par rapport au système de la langue, tous les enseignants observés demandent aux élèves de répertorier différentes catégories de procédures utilisées pour reprendre les personnages d'un récit. L'un ou l'autre participant fait un travail approfondi sur les caractéristiques grammaticales de ces reprises. Certaines consignes témoignent aussi de 
la volonté de réviser certaines connaissances grammaticales construites antérieurement dans des leçons de grammaire de la phrase.

L'institutionnalisation de l'objet « reprises anaphoriques » sur le plan du système de la langue est plus rare. Seule l'enseignante de $7-8^{\mathrm{H}}\left(\mathrm{CM} 2-6^{\mathrm{e}}\right.$ en France) se distingue des autres enseignants en proposant plusieurs institutionnalisations en lien avec le système langagier dans lequel s'insèrent les reprises anaphoriques.

Enfin, tous les participants proposent une consigne centrée sur le style volontairement ambigu de certains auteurs (annexes 1 et 2 ) ou la comparaison entre deux textes d'auteurs au style différent. Deux enseignants seulement institutionnalisent cette dimension.

\section{Les outils langagiers utilisés pour parler de l'objet « reprises anaphoriques » dans les consignes et les institutionnalisations}

Pour parler de l'objet enseigné "reprises anaphoriques », les enseignants emploient différents outils langagiers que nous avons cherché à pointer (tableau 4).

Tableau 4. Procédés langagiers utilisés par les enseignants pour parler de la désignation des personnages dans les consignes et institutionnalisations.

\begin{tabular}{|c|c|c|c|c|c|c|c|c|c|}
\hline \multirow{2}{*}{$\begin{array}{l}\text { N. de dispositifs } \\
\text { d'institution-nalsation }\end{array}$} & & \multicolumn{8}{|c|}{ Procédés langagiers utilisés pour parler de l’objet } \\
\hline & & $\begin{array}{c}\text { Pronom } \\
\text { démonstratif }\end{array}$ & $\begin{array}{c}\text { Métalangage } \\
\text { syntaxique }\end{array}$ & $\begin{array}{c}\text { Métalangage } \\
\text { textuel }\end{array}$ & "Mot " & $\begin{array}{l}\text { Substitution } \\
\text { lexicale }\end{array}$ & Périphrase & $\begin{array}{l}\text { Autre } \\
\text { pronom }\end{array}$ & TOTAL \\
\hline \multirow[t]{2}{*}{57} & Dans les consignes & 11 & 40 & 2 & 33 & 20 & 105 & 3 & 214 \\
\hline & & $5 \%$ & $19 \%$ & $1 \%$ & $15.5 \%$ & $9.5 \%$ & $49 \%$ & $1 \%$ & \\
\hline \multirow[t]{4}{*}{31} & Dans les & 52 & 19 & 2 & 16 & 22 & 27 & 15 & 153 \\
\hline & & $34 \%$ & $12.5 \%$ & $1 \%$ & $10,5 \%$ & $14,5 \%$ & $17.5 \%$ & $10 \%$ & \\
\hline & Dans les consignes et & 63 & 59 & 4 & 49 & 42 & 132 & 18 & 367 \\
\hline & & $17 \%$ & $16 \%$ & $1 \%$ & $13.5 \%$ & $11.5 \%$ & $36 \%$ & $5 \%$ & \\
\hline
\end{tabular}

Analyser plus précisément comment ces enseignants parlent du savoir à construire, dans les consignes et les institutionnalisations, met en évidence les mots que les élèves doivent interpréter pour construire leurs propres connaissances. Nous remarquons dans le tableau ci-dessus que des pronoms démonstratifs servent souvent à nommer l'objet « reprises anaphoriques ». Les enseignants emploient aussi d'autres procédés langagiers : des mots issus d'un métalangage grammatical, des substitutions lexicales diverses et des périphrases.

Les pronoms démonstratifs sont des reprises qui servent surtout à institutionnaliser et qui s'insèrent dans un discours et un environnement didactique plus large. Des pronoms tels que «ça » ou « c'est » mettent le doigt sur l'objet institutionnalisé ; si ces déictiques sont par essence vagues et peu précis, ils font toujours référence à autre chose que les enseignants pointent à l'aide de ce procédé (par exemple une liste de reprises au tableau noir).

Pour désigner les reprises anaphoriques, les enseignants s'appuient aussi fortement sur un vocabulaire construit dans les leçons de grammaire de la phrase. Les termes grammaticaux "nom, pronom, groupe nominal, et plus rarement adjectif, synonyme " sont régulièrement utilisés; ils font référence à des notions syntaxiques ou lexicales, et 
non à des notions textuelles. Dans les consignes tout comme dans les institutionnalisations, un métalangage textuel, par exemple "reprises anaphoriques", «cohésion nominale », « reprises » ou encore «désignations », n'est presque jamais utilisé6. périphrases pour pointer l'objet enseigné (tableau ci-avant, 105/214). Selon S. Chartrand, D. Aubin, R. Blain et C.Simard (1999:31), la périphrase est une forme de reprise nominale, constituée d'une «suite de mots utilisés pour désigner une réalité qu'un seul mot pourrait nommer ", en lui apportant un supplément d'information. Lorsqu'on observe les exemples ci-après, on remarque la richesse d'un tel procédé : « un mot qui représente un des personnages », " tous les autres mots que vous avez utilisés pour dire la maitresse ", « les mots que vous avez utilisés pour désigner les personnages », " la variété des mots qu'on utilise pour parler de chacun des personnages », " des moyens de donner des informations au lecteur et d'éviter de répéter plusieurs fois la même chose ». Par la périphrase, les enseignants observés pointent clairement l'objet d'enseignement en employant les verbes "désigner ", " reprendre » ou "nommer »; de plus, ils mettent l'accent sur la fonction de ces désignations : rendre les textes plus intéressants, parler de chacun des personnages, qualifier les personnages, donner des informations au lecteur ou encore éviter de répéter plusieurs fois le même mot.

pouvons tenter des hypothèses quant à l'intention, peut-être inconsciente, soustendue par ce procédé langagier, visible surtout dans les consignes. Nous remarquons que par la périphrase, les enseignants emploient un métalangage textuel ou un synonyme mais sous leur forme verbale (reprendre, désigner, appeler, parler de, qualifier), tout en insistant sur les fonctions des reprises anaphoriques dans la construction du récit et plus particulièrement dans la construction des personnages. Aussi, ce procédé est plus riche qu'il n'y parait. Il ne remplace pas la terminologie que l'on retrouve dans les grammaires de référence et les plans d'études, mais il capte peut-être davantage l'attention de l'élève en lui parlant du savoir à construire avec des mots qu'il comprend.

\section{Conclusion}

Par cet article, nous avons décrit des pratiques d'enseignement de l'écriture, pratiques qui tentent des liens entre écriture et construction de connaissances ciblées sur un genre spécifique. Il montre l'intérêt de telles pratiques pour l'enseignement de l'écriture, mais aussi pour donner du sens aux apprentissages grammaticaux et les réinvestir en contexte. 
Aussi, si de telles pratiques permettent de faire des liens - que nous jugeons essentiels entre développement des capacités d'écriture et savoir grammatical, elles ne semblent pas suffisantes pour construire des apprentissages grammaticaux explicites; en effet, dans de telles pratiques, les connaissances grammaticales disparaissent quelque peu. $\mathrm{Ce}$ constat rejoint les propos de S. Toulou et B. Schneuwly (2009: 326) : «Pour la grammaire comme description et connaissance, être au service de l'expression [...] se paie, pourraiton dire, au prix de sa propre disparition ». Lorsque les enseignants utilisent la grammaire à des fins d'expression, c'est bien pour amener l'élève à mieux écrire et non pour construire des connaissances sur la langue. Aussi, si l'on veut pérenniser les démarches d'analyse descriptive et la construction de connaissances explicites sur la langue et la communication, il est à la fois nécessaire de maintenir un enseignement de la grammaire pour elle-même et viser son intégration à l'action langagière par des dispositifs intégratifs telle la séquence didactique proposée dans cette recherche.

Enfin, nous pensons que le développement de gestes didactiques efficaces, notamment les gestes de régulation et d'institutionnalisation, doit devenir une préoccupation pour les formateurs en didactique du français, car ce sont ces gestes qui font de l'objet un objet enseigné et c'est par eux que l'apprentissage des élèves peut, peut-être, se réaliser.

\section{BIBLIOGRAPHIE}

ALLAL, L. (2007) : « Régulations des apprentissages : orientations conceptuelles pour la recherche et la pratique en éducation », in : L. Mottier Lopez \& L. Allal (éds), Régulation des apprentissages en situation scolaire et en formation, Bruxelles, De Boeck, p. 8-23.

BRONCKART, J.-P. (1996a) : Activité langagière, textes et discours, Neuchâtel/Paris, Delachaux \& Niestlé.

- (1996b) : «Genres de textes, types de discours et opérations psycholinguistiques », Enjeux, 37/38, p. 31-47.

- (2004) : Didactique de la grammaire, Genève, Département de l'instruction publique, Secteur langues et cultures.

BUCHETON, D. (1994) : « Décloisonner ou comment mettre la langue, le langage, le texte à distance ", Le français aujourd'hui, 107, p. 60-65.

Brousseau, G. (1998) : Théorie des situations didactiques, Grenoble, Éd. La Pensée sauvage.

CIIP (2006) : Enseignement/apprentissage du français en Suisse romande : orientations. Neuchâtel, conférence intercantonale de l'instruction publique de la Suisse romande et du Tessin.

- (2010) : Plan d'études romand, Neuchâtel, conférence intercantonale de l'instruction publique de la Suisse romande et du Tessin.

CHABANNE, J.-C. (2004) : « La grammaire est-elle entièrement soluble dans les pratiques

d'écriture ? », in : C. Vargas (éd.), Langue et étude de la langue : approches linguistiques et didactiques, Aix-en-Provence, Presses universitaires de Provence, p. 125-134. 
CHARTRAND, S. (1996) : Pour un nouvel enseignement de la grammaire : propositions didactiques, $2^{\mathrm{e}}$ éd., Montréal, Éd. logiques.

CHARTRAND, S. \& BOIVIN, M.-C. (2004) : Articulation des activités métalinguistiques aux activités discursives dans la classe de français au secondaire inférieur, actes du $9^{\mathrm{e}}$ colloque de l'Association internationale pour la recherche en didactique du français (AIRDF), Québec, Université Laval.

CHARTRAND, S., AUBIN, D. BLAIN, R. \& SIMARD, C. (1999) : Grammaire pédagogique du français d'aujourd'hui, Montréal, Graficor.

CLOT, Y., FAÏTA, D. \& SCHELLER, L. (2000) : « Entretiens en autoconfrontation croisée : une méthode en clinique de l'activité », Pistes. Réflexion sur la pratique, 2(1) (en ligne : http://

pistes.revues.org/3833, consulté le 03/05/09).

combettes, B. (1988) : Pour une grammaire textuelle, Bruxelles, De Boeck/Duculot.

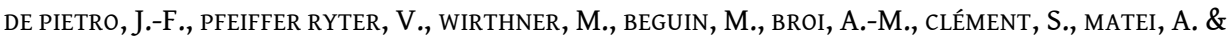
ROOS, E. (2009) : Évaluation du moyen d'enseignement « S'exprimer en français » : rapport final, Neuchâtel, IRDP.

DIP (1996) : Programme de français de 2P à 6P, Fribourg, Département de l'instruction publique du canton de Fribourg, enseignement primaire.

DOLZ, J., SCHNEUWLY, B. \& NOVERRAZ, M. (2001) : S'exprimer en français. Séquences didactiques pour l'oral et l'écrit, Bruxelles, De Boeck.

GoIgoux, R. (2007) : « Un modèle d'analyse de l'activité des enseignants », Éducation \& Didactique, vol. 1 (3), p. 47-70.

MARMY CUSIN, V. (2012) : Développer et comprendre des pratiques d'enseignement de la grammaire, intégrées à la production textuelle : entre les dires et les faires, thèse de doctorat en sciences de l'éducation, université de Genève (en ligne : http://archive-ouverte.unige.ch/vital/access/ manager/Repository/unige:20294, consulté le 30/10/13).

MARMY CUSIN, V. \& SCHNEUWLY, B. (2013) : «Intégrer des apprentissages grammaticaux à l'étude d'un genre textuel : entre les dires et les faires », in : O. Bertrand \& I. Schaffner (dirs), Enseigner la grammaire, Paris/Pallaiseau, Éd. de l'École polytechnique, p. 357-375.

MAINGUENEAU, D. (2005) : « Réflexions sur la "grammaire du discours" au collège », Le français aujourd'hui, 148, p. 47-54.

SCHNEUWLY, B. \& DOLZ, J. (1997) : « Les genres scolaires : des pratiques langagières aux objets d'enseignement ", Repères, 15, p. 27-41.

- (2009) : Des objets enseignés en classe de français : le travail de l'enseignant sur la rédaction de textes argumentatifs et sur la subordonnée relative, Rennes, Presses universitaires de Rennes.

SCHNEUWly, B. \& Toulou, S. (2009) : "Deux visées complémentaires : les dispositifs didactiques », in : B. Schneuwly \& J. Dolz (éds), Des objets enseignés en classe de français : le travail de l'enseignant sur la rédaction de textes argumentatifs et sur la subordonnée relative, Rennes, PUR, p. 309-327.

Sensevy, G. (2007) : « Des catégories pour décrire et comprendre l'action didactique », in : G. Sensevy \& A. Mercier (éds), Agir ensemble, Rennes, PUR, p. 13-49.

Tisset, C. \& Léon, R. (1992) : Enseigner le français à l'école, Paris, Hachette. 


\section{NOTES}

1. Au moment de la recherche (2009), les plans d'études en vigueur (CIIP, 1989 ; DIP, 1996) ne demandent pas un travail spécifique en production sur la cohésion nominale. Seul le plan d'études cantonal fait mention de cet objet dans le domaine de la compréhension («établir une relation entre des mots et leur(s) reprise(s)», DIP, 1996: 7). En revanche, les moyens officiels romands (Dolz et al., 2001) traitent régulièrement de cet objet dans le domaine de la production orale et écrite. De même, le nouveau plan d'études romand (CIIP, 2010), en consultation au moment de la recherche, prend en compte cet aspect dans le domaine de la production (écriture d'un texte en "respectant l'utilisation de reprises pronominales et nominales", p. 34), de la compréhension ("repérage des différents termes désignant un même personnage », p. 24) et du fonctionnement de la langue («Compréhension des reprises anaphoriques », p. 70).

2. Si les discours à propos de l'action intéressent le lecteur, voir V. Marmy Cusin et B. Schneuwly (2013).

3. Trois classes de 5-6H (CE2-CM1 en France) et une classe de 7-8H (CM2-6e).

4. Un dispositif didactique est concrètement mis en œuvre par une consigne et la création de conditions concrètes qui définissent une activité scolaire à effectuer par les élèves. Il y a changement de dispositif lorsque l'objet enseigné change ou que l'activité proposée se modifie (nouvelle consigne, nouveau support matériel ou nouvelle forme de travail) (Schneuwly \& Dolz, 2009).

5. Schémas réalisés à partir du logiciel Transana.

6. Dans les plans d'études en Suisse romande (DIP, 1996 ; CIIP, 1989, 2010), les termes de reprises, de reprises anaphoriques, de reprises nominales et pronominales et de termes désignant un même personnage font référence à la cohésion nominale d'un texte.

\section{RÉSUMÉS}

Dans cet article, l'auteure décrit des pratiques d'enseignement de l'écriture qui favorisent les liens entre écriture et construction de connaissances ciblées sur un genre spécifique. Une première partie définit le genre textuel comme point d'appui à l'enseignement de l'écriture ainsi qu'à l'articulation entre écriture et apprentissage grammatical. Par la suite, l'auteure présente les caractéristiques méthodologiques d'une recherche doctorale (Marmy Cusin, 2012), centrée sur la pratique d'enseignants au niveau de l'école primaire en Suisse romande mettant en œuvre une séquence didactique articulant grammaire et texte. La dernière partie de l'article présente quelques résultats extraits de cette recherche : les textes d'auteurs et les textes des élèves sont au cœur des dispositifs mis en œuvre par les enseignants observés. La notion textuelle travaillée dans la séquence didactique (cohésion des chaines référentielles désignant les personnages d'un récit) n'est pas facile à conceptualiser au niveau primaire et nécessite une médiation importante de la part des enseignants. Enfin, l'enseignement grammatical comme description du fonctionnement de la langue et de la communication est peu visible dans ces pratiques articulées : l'enseignement de l'écriture prend le pas sur l'enseignement de la grammaire. Ce dernier constat laisse à penser que si l'on veut pérenniser les démarches d'analyse descriptive et la construction de connaissances explicites sur la langue et la communication, il est à la fois 
nécessaire de maintenir un enseignement de la grammaire pour elle-même et viser son intégration à l'action langagière par des dispositifs intégratifs telle la séquence didactique proposée dans cette recherche.

In this paper, the author describes practices for the teaching of writing, which enhance the links between writing and knowledge construction centred on a specific text genre. The first part of this paper defines the notion of text genre as a tool for the teaching of writing as well as a way to link the latter with grammar learning. The author goes on to present the methodological characteristics of her doctoral research (Marmy Cusin, 2012), which focuses on teachers' practices at primary level in French-speaking Switzerland. These teachers experiment a didactic sequence articulating grammar and writing. The last part of the paper presents some of the significant results of this research: authors' texts and pupils' texts are at the heart of practices developed by the observed teachers. The textual notion studied here (cohesive relations) is not easy to conceptualize at primary level and requires an important mediation role on the part of the teachers. The teaching of grammar is scarcely visible in these articulated practices: the teaching of writing takes over the teaching of grammar. If one is to develop descriptive analysis procedures and explicit knowledge on language and communication, the author argues therefore that it is both necessary to maintain the teaching of explicit grammar and to integrate it to wider language proficiency through the use of articulated practices such as the didactic sequence proposed in this research.

\section{INDEX}

Mots-clés : écriture, genre textuel, articulation entre production textuelle et apprentissage grammatical, pratique enseignante, enseignement au niveau primaire

Keywords : teaching of writing, text genre, didactic sequence articulating grammar and writing, teachers' practices at primary level

\section{AUTEUR}

\section{VÉRONIQUE MARMY CUSIN}

GRAFE, HEP-Fribourg, université de Genève 